\title{
QED logarithms in the electroweak corrections to the muon anomalous magnetic moment
}

\author{
G. Degrassi* and G. F. Giudice ${ }^{\dagger}$ \\ Theory Division, CERN, CH-1211 Geneva 23, Switzerland \\ (Received 18 March 1998; published 5 August 1998)
}

\begin{abstract}
We employ an effective Lagrangian approach to derive the leading-logarithm two-loop electroweak contributions to the muon anomalous magnetic moment, $a_{\mu}$. We show that these corrections can be obtained using known results on the anomalous dimensions of composite operators. We confirm the result of Czarnecki et al. for the bosonic part and present the complete $\sin ^{2} \theta_{W}$ dependence of the fermionic contribution. The approach is then used to compute the leading-logarithm three-loop electroweak contribution to $a_{\mu}$. Finally we derive, in a fairly model-independent way, the QED improvement of new-physics contributions to $a_{\mu}$ and to the electric dipole moment (EDM) of the electron. We find that the QED corrections reduce the effect of new physics at the electroweak scale by $6 \%$ (for $a_{\mu}$ ) and by $11 \%$ (for the electron EDM). [S0556-2821(98)03017-3]
\end{abstract}

PACS number(s): 13.40.Em, 12.15.Lk, 14.60.Ef

\section{INTRODUCTION}

The measurement of the anomalous magnetic moment of the muon [1],

$$
a_{\mu} \equiv \frac{g_{\mu}-2}{2} \equiv \frac{\mu_{\mu}}{\left(e \hbar / 2 m_{\mu}\right)}-1=\left(\begin{array}{lll}
11 & 659 & 230 \pm 84
\end{array}\right) \times 10^{-10} \text {, }
$$

provides both a test of the validity of QED [2] and a probe of possible extensions of the standard model (SM) electroweak theory. From the experimental point of view, the E821 experiment at Brookhaven National Laboratory is expected to improve the accuracy in the $a_{\mu}$ measurement to the level of $4 \times 10^{-10}$, and possibly to $1-2 \times 10^{-10}$ if large statistics is accumulated [3]. Let us consider now the present status of the theoretical prediction for $a_{\mu}$. It is convenient to separate the total result into several different parts. The pure QED contribution, which is known to order $\alpha^{5}$ [2], is

$$
a_{\mu}^{\mathrm{QED}}=(116584706 \pm 2) \times 10^{-11} .
$$

The part affected by strong-interaction contributions, which contains the largest source of uncertainty, comes from the hadron vacuum polarization and the hadronic light-by-light amplitude. A recent analysis relating, by means of dispersion relations, the hadronic vacuum polarization to data from $e^{+} e^{-}$annihilation and $\tau$ decays gives [4]

$$
a_{\mu}^{\mathrm{had}}(\operatorname{vac} \mathrm{pol})=(6951 \pm 75) \times 10^{-11} \text {. }
$$

The error is dominated by the experimental uncertainty and will be significantly improved by future measurements at the Beijing Electron-Positron Collider (BEPC), at DAФNE in Frascati, and at VEPP-2M in Novosibirsk. The effects of higher-order hadronic contributions have also been evaluated [5]:

\footnotetext{
*Permanent address: Dipartimento di Fisica, Università di Padova, Padova, Italy.

${ }^{\dagger}$ On leave of absence from INFN, Sezione di Padova, Italy.
}

$$
a_{\mu}^{\text {had }}(\text { h.o. vac pol })=(-101 \pm 6) \times 10^{-11}
$$

The most recent theoretical estimate of the hadronic light-bylight contribution gives [6]

$$
a_{\mu}^{\mathrm{had}}(\gamma \times \gamma)=(-79 \pm 15) \times 10^{-11} .
$$

Finally, the SM electroweak contribution at one loop is [7]

$$
a_{\mu}^{\mathrm{EW}}(1 \text { loop })=\frac{5 G_{\mu} m_{\mu}^{2}}{24 \sqrt{2} \pi^{2}}\left[1+\frac{1}{5}\left(1-4 s_{W}^{2}\right)^{2}\right]=195 \times 10^{-11},
$$

where $G_{\mu}$ is the Fermi constant and $s_{W}^{2} \equiv \sin ^{2} \theta_{W}=1$ $-M_{W}^{2} / M_{Z}^{2}$. As first noticed in Ref. [8], two-loop electroweak corrections are quite substantial, because of large contributions $O\left(G_{\mu} m_{\mu}^{2} \alpha / \pi \ln \left(M / m_{f}\right)\right)$. Here $M$ represents the $W$ or $Z$ mass and $m_{f}$ indicates a light fermion mass. Combining together the contribution containing closed fermionic loops $[9,10]$ and the one from the other relevant two-loop diagrams (usually indicated as the bosonic part), Czarnecki et al. obtain $[11]$

$$
a_{\mu}^{\mathrm{EW}}(2 \text { loop })=(-44 \pm 4) \times 10^{-11},
$$

for a Higgs boson mass $M_{H}=250 \mathrm{GeV}$, where the error is associated with the uncertainties in $M_{H}$, the hadronic contributions, and higher-loop effects.

In this paper, we show how the leading $O\left(G_{\mu} m_{\mu}^{2} \alpha / \pi \ln \left(M / m_{f}\right)\right)$ two-loop electroweak corrections to the anomalous magnetic moment, $a_{\mu}^{\mathrm{EW}}(2 \text { loop })_{L L}$, can be easily obtained with the help of effective theories and the Wilson renormalization group. The relevant anomalous dimensions of composite operators can be extracted from known results of the QCD corrections to flavor-violating bottom-quark transitions mediated by the magnetic dipole operator [12]. In this way, we derive $a_{\mu}^{\mathrm{EW}}(2 \text { loop })_{L L}$ without directly computing any Feynman diagram and confirm the results of Refs. [9-11]. Moreover, since the renormalizationgroup technique actually includes all leading QED logarithms, we are able to give an analytic expression for the leading three-loop contribution to $a_{\mu}^{\mathrm{EW}}$. This contribution 
turns out to be very small, but its knowledge allows us to eliminate the uncertainty in $a_{\mu}^{\mathrm{EW}}$ from higher-order effects. Including the different terms, we obtain

$$
a_{\mu}^{\mathrm{EW}}=(153 \pm 3) \times 10^{-11},
$$

where the central value corresponds to $M_{H}=150 \mathrm{GeV}$. This leads to a theoretical prediction for the muon anomalous magnetic moment:

$$
a_{\mu}=\left(\begin{array}{lll}
116591630 \pm 77
\end{array}\right) \times 10^{-11} .
$$

We also show that this method is well suited to compute higher-order corrections to new-physics contributions to magnetic and electric dipole moments. The only necessary basic assumption is that the new physics gives a one-loop contribution to the dipole moments, but does not significantly affect at the tree level composite four-fermion interactions. Under this assumption, the leading logarithmic twoloop contribution is determined by infrared effects and it can be computed from the renormalization-group evolution of the effective theory below the weak scale. We find that the QED improvement reduces the one-loop new-physics effect by $6 \%$ in the case of $a_{\mu}$ and $11 \%$ in the case of the electric dipole moment (EDM) of the electron, assuming that the new physics lies around the weak scale.

\section{TWO-LOOP CALCULATION OF $\boldsymbol{a}_{\mu}^{\mathrm{EW}}$}

The leading two-loop contributions to $a_{\mu}^{\mathrm{EW}}$ come from large QED logarithms. These terms correspond to ultraviolet divergences in the effective theory obtained by integrating out the heavy modes, and they can be derived in terms of the anomalous dimensions of the dipole and current-current operators.

We start by defining an effective theory valid below the electroweak scale, in which the $W$ and $Z$ bosons and the top quark have been integrated out. The effect from heavy particles is reflected in higher-dimensional operators. Here we are interested in the operator corresponding to $a_{\mu}$,

$$
H_{\mu}=-\sqrt{\frac{\alpha}{(4 \pi)^{3}}} m_{\mu} \bar{\mu} \sigma^{\nu \rho} \mu F_{\nu \rho}
$$

and to other possible dimension-six operators that mix under QED renormalization with $H_{\mu}$. Since QED is parity conserving and $H_{\mu}$ is parity even, it is easy to realize that we need to consider only the following parity-even four-fermion operators:

$$
\begin{gathered}
V_{\mu}=\frac{1}{2} \bar{\mu} \gamma^{\nu} \mu \bar{\mu} \gamma_{\nu} \mu, \quad A_{\mu}=\frac{1}{2} \bar{\mu} \gamma^{\nu} \gamma_{5} \mu \bar{\mu} \gamma_{\nu} \gamma_{5} \mu, \\
V_{\mu f}=\bar{\mu} \gamma^{\nu} \mu \bar{f} \gamma_{\nu} f, \quad A_{\mu f}=\bar{\mu} \gamma^{\nu} \gamma_{5} \mu \bar{f} \gamma_{\nu} \gamma_{5} f .
\end{gathered}
$$

In Eq. (12), $f$ indicates a generic fermion different from $\mu$ and the factor $1 / 2$ in the definition of the operators $V_{\mu}$ and $A_{\mu}$ compensates the symmetry factor for two identical currents in the Feynman rule. The relevant part of the effective Lagrangian is

$$
\mathcal{L}_{\mathrm{eff}}=-2 \sqrt{2} G_{\mu} \sum_{i} C_{i} \mathcal{O}_{i}
$$

where the operators $\mathcal{O}_{i}$ are given in Eqs. (10)-(12) (with $f$ $=\{b, \tau, c, s, d, u, e\})$ and the Wilson coefficients $C_{i}$ at the electroweak scale $\mu=M$ are

$$
\begin{gathered}
C_{H_{\mu}}(M)=\frac{2 \sqrt{2} \pi^{2}}{G_{\mu} m_{\mu}^{2}} a_{\mu}^{\mathrm{EW}}(1 \text { loop })=\frac{5}{12}\left[1+\frac{1}{5}\left(1-4 s_{W}^{2}\right)^{2}\right] \\
C_{V_{\mu}}(M)=\frac{1}{8}\left(1-4 s_{W}^{2}\right)^{2}, \quad C_{A_{\mu}}(M)=\frac{1}{8} \\
C_{V_{\mu f}}(M)=-\frac{1}{4}\left(1-4 s_{W}^{2}\right)\left(T_{f}-2 Q_{f}^{2} s_{W}^{2}\right) \\
C_{A_{\mu f}}(M)=-\frac{1}{4} T_{f}
\end{gathered}
$$

In Eq. (16), $T_{f}$ and $Q_{f}$ are the third isospin component and electric charge of the fermion $f$, respectively. The coefficient $C_{H_{\mu}}$ is obtained by one-loop integration, while the coefficients in Eqs. (15) and (16) correspond to tree-level $Z$ exchange. The $W$ boson can only generate operators with $f$ $=\nu$ which are irrelevant for our analysis, because they cannot mix under QED with $H_{\mu}$ as neutrinos carry no electric charge.

We are interested in the Wilson coefficient of the operator $H_{\mu}$ at the scale $\mu=m_{\mu}$. The leading-logarithmic evolution of the Wilson coefficients from the scale $M$ to a generic scale $\mu$ is given in terms of the one-loop anomalous dimension matrix $\gamma$ and the one-loop beta function. In our case,

$$
\begin{aligned}
C_{i}(\mu) & =\sum_{j}\left[\exp \int_{e(M)}^{e(\mu)} d e \frac{\gamma^{T}(e)}{\beta(e)}\right]_{i j} C_{j}(M) \\
& =\sum_{j}\left\{V\left[\frac{\alpha(M)}{\alpha(\mu)}\right]^{\hat{\gamma} / 2 b} V^{-1}\right\}_{i j} C_{j}(M),
\end{aligned}
$$

where $e=\sqrt{4 \pi \alpha}$ is the QED gauge coupling, and the rotation matrix $V$ is defined such that $\hat{\gamma}=V^{-1} \gamma^{T} V$ is diagonal. The beta function is $\beta(e)=-b e^{3} /\left(16 \pi^{2}\right)$, with the coefficient $b$ given by

$$
b=-\frac{4}{3} \sum_{f} N_{f} Q_{f}^{2}
$$

In Eq. (18) the sum is extended over all fermions $f$ with mass less than the scale $\mu$, electric charge $Q_{f}$ and multiplicity $N_{f}\left(N_{f}=3\right.$ for quarks and $N_{f}=1$ for leptons). Expanding Eq. (17) in powers of $\alpha$, we obtain

$$
C_{H_{\mu}}(\mu)=C_{H_{\mu}}(M)-\sum_{i} \gamma\left(\mathcal{O}_{i}, H_{\mu}\right) \frac{\alpha(\mu)}{4 \pi} \ln \frac{M}{\mu} C_{\mathcal{O}_{i}}(M),
$$


where $\gamma\left(\mathcal{O}_{i}, H_{\mu}\right) \equiv \gamma_{\mathcal{O}_{i} H_{\mu}}$. Aside from an overall factor $G_{\mu} m_{\mu}^{2} /\left(2 \sqrt{2} \pi^{2}\right)$, the first term on the right-hand side (RHS) of Eq. (19) gives $a_{\mu}^{\mathrm{EW}}$ (1 loop), while the second term gives $a_{\mu}^{\mathrm{EW}}(2 \text { loop })_{L L}$.

The last ingredient necessary to complete the analysis is the computation of the elements $\gamma\left(\mathcal{O}_{i}, H_{\mu}\right)$ of the anomalous dimension matrix. This requires a calculation of the divergent parts of loop diagrams generating $H_{\mu}$, in which a single operator $\mathcal{O}_{i}$ is inserted and a single photon is exchanged. Actually, this calculation is completely analogous to the one of the QCD anomalous dimension matrix for the $\Delta B=1$ effective Lagrangian, relevant for flavor-violating bottomquark transitions. For such a processes a complete list of the divergent contributions from the various diagrams can be found in Ref. [13]. All we need to do is the proper translation from quarks to muons, and from gluons to photons. In this way we obtain

$$
\gamma\left(H_{\mu}, H_{\mu}\right)=16
$$

$$
\begin{gathered}
\gamma\left(V_{\mu}, H_{\mu}\right)=\frac{40}{3} \\
\gamma\left(A_{\mu}, H_{\mu}\right)=\frac{808}{9} \\
\gamma\left(V_{\mu f}, H_{\mu}\right)=\frac{32}{9} Q_{f}^{2} N_{f} \\
\gamma\left(A_{\mu f}, H_{\mu}\right)=48 Q_{f}^{2} N_{f} .
\end{gathered}
$$

These expressions are valid both in dimensional regularization with the 't Hooft-Veltman prescription for $\gamma_{5}[14]$ and in dimensional reduction [15]. In both schemes there are no finite operator renormalizations from non-vanishing matrix elements.

Replacing in Eq. (19) the matching conditions of Eqs. (14)-(16) and the anomalous-dimension elements of Eqs. (20)-(24), and choosing $M=M_{Z}$ as the high-energy scale, we obtain

$$
\begin{aligned}
a_{\mu}^{\mathrm{EW}}(2 \text { loop })_{L L}= & \frac{5 G_{\mu} m_{\mu}^{2}}{24 \sqrt{2} \pi^{2}} \frac{\alpha\left(m_{\mu}\right)}{\pi}\left\{-\frac{43}{3}\left[1+\frac{31}{215}\left(1-4 s_{W}^{2}\right)^{2}\right] \ln \frac{M_{Z}}{m_{\mu}}\right. \\
& \left.+\frac{36}{5} \sum_{f \in F} N_{f} Q_{f}^{2}\left[T_{f}+\frac{2}{27}\left(T_{f}-2 Q_{f} s_{W}^{2}\right)\left(1-4 s_{W}^{2}\right)\right] \ln \frac{M_{Z}}{m_{f}}\right\},
\end{aligned}
$$

where the sum is extended over the fermions with a mass threshold between $M_{Z}$ and $m_{\mu}, F=\{b, \tau, c, s, d, u\}$. Equation (25) confirms the results of Refs. [9-11], but it disagrees with the one presented in Ref. [8]. It also shows the complete $s_{W}^{2}$ dependence, extending the results of Refs. $[9,10]$, in which the fermionic contribution has been computed in the limit $s_{W}^{2}=1 / 4$.

The contribution from light quarks is not appropriately described by Eq. (25), since perturbation theory is not justified. Nevertheless, here we will parametrize the effect by taking in Eq. (25) $m_{Q} \equiv m_{u}=m_{d}=m_{s}=0.3 \mathrm{GeV}$, and varying $m_{Q}$ between 0 and $1 \mathrm{GeV}$. In this way, our result is consistent with the estimate of the light-quark contribution given in Ref. [10], based on a chiral effective Lagrangian. Taking $m_{c}=1.5 \mathrm{GeV}$ and $m_{b}=4.5 \mathrm{GeV}$, we find $a_{\mu}^{\mathrm{EW}}(2 \text { loop })_{L L}=-(37 \pm 1) \times 10^{-11}$. This corresponds to $\sim 19 \%$ of the one-loop contribution. We recall that the result in Eq. (25) includes only the logarithmic contribution. The terms not enhanced by large logarithms have been computed in Refs. $[9,11]$ and amount to $a_{\mu}^{\mathrm{EW}}(2 \text { loop })_{N L}=-(6 \pm 2)$ $\times 10^{-11}$, where the central value corresponds to a Higgs boson mass $M_{H}=150 \mathrm{GeV}$ and the error to a variation of $M_{H}$ in the range between 100 and $1000 \mathrm{GeV}$.

\section{THREE-LOOP CALCULATION OF $a_{\mu}^{\mathrm{EW}}$}

In the previous section, we have computed $a_{\mu}^{\mathrm{EW}}(2 \text { loop })_{L L}$ by expanding Eq. (17) at the first order in $\alpha$. However, Eq. (17) also contains the information of higher-order terms, since it resums all leading logarithms. We can therefore easily evaluate the magnitude of higher-order corrections. For this purpose it is sufficient to expand Eq. (17) to the next order in $\alpha$, because $(\alpha / \pi) \ln \left(M_{Z} / m_{\mu}\right)$ is much smaller than 1 . The corresponding result will give us the leading logarithmic part of $a_{\mu}^{\mathrm{EW}}$ (3 loop).

Retaining $\mathcal{O}\left(\alpha^{2}\right)$ terms, the expansion of Eq. (17) yields

$$
\begin{aligned}
C_{i}(\mu)= & \sum_{j}\left\{\delta_{i j}-\gamma_{j i} \frac{\alpha(\mu)}{4 \pi} \ln \frac{M}{\mu}\right. \\
& \left.+\left[b \gamma_{j i}+\frac{1}{2}(\gamma \gamma)_{j i}\right]\left[\frac{\alpha(\mu)}{4 \pi} \ln \frac{M}{\mu}\right]^{2}\right\} C_{j}(M) .
\end{aligned}
$$

Because of the presence of the $(\gamma \gamma)$ factor, we now need information on the complete structure of the anomalous dimension matrix, and not only on the elements given in Eqs. (20)-(24). We start by defining the basis for the required 
parity-even operators. In addition to the operators defined in Eqs. (10)-(12), we need the following four-fermion operators:

$$
\begin{gathered}
V_{f}=\frac{1}{2} \bar{f} \gamma^{\nu} f \bar{f} \gamma_{\nu} f, \quad A_{f}=\frac{1}{2} \bar{f} \gamma^{\nu} \gamma_{5} f \bar{f} \gamma_{\nu} \gamma_{5} f, \\
V_{f f^{\prime}}=\bar{f} \gamma^{\nu} f \bar{f}^{\prime} \gamma_{\nu} f^{\prime}, \quad A_{f f^{\prime}}=\bar{f} \gamma^{\nu} \gamma_{5} f \bar{f}^{\prime} \gamma_{\nu} \gamma_{5} f^{\prime} \quad \text { with } f \neq f^{\prime},
\end{gathered}
$$

$\widetilde{V}_{q q^{\prime}}=\bar{q} \gamma^{\nu} q^{\prime} \bar{q}^{\prime} \gamma_{\nu} q, \quad \widetilde{A}_{q q^{\prime}}=\bar{q} \gamma^{\nu} \gamma_{5} q^{\prime} \bar{q}^{\prime} \gamma_{\nu} \gamma_{5} q$ with $q \neq q^{\prime}$.

In Eqs. (27)-(29), $f, f^{\prime}\left(q, q^{\prime}\right)$ represent a generic fermion (quark), and all quark operators are defined with the color indices saturated so that each current is an $S U(3)_{C}$ singlet. The operators $\widetilde{V}_{q q^{\prime}}$ and $\widetilde{A}_{q q^{\prime}}$ cannot be written in terms of $V_{f f^{\prime}}$ and $A_{f f^{\prime}}$ with a Fierz rearrangement, because of the different color-index saturation.

The matching conditions for the Wilson coefficients at the scale $\mu=M$ are

$$
\begin{gathered}
C_{V_{f}}(M)=\frac{1}{2}\left(T_{f}-2 Q_{f} s_{W}^{2}\right)^{2}, \quad C_{A_{f}}(M)=\frac{1}{2} T_{f}^{2}, \\
C_{V_{f f^{\prime}}}(M)=\frac{1}{2}\left(T_{f}-2 Q_{f} s_{W}^{2}\right)\left(T_{f^{\prime}}-2 Q_{f^{\prime}} s_{W}^{2}\right), \\
C_{A_{f f^{\prime}}}(M)=\frac{1}{2} T_{f} T_{f^{\prime}}, \\
C_{\widetilde{V}_{q q^{\prime}}}(M)=C_{\tilde{A}_{q q^{\prime}}}(M)=\frac{1}{4} \Delta_{q q^{\prime}},
\end{gathered}
$$

where $\Delta_{q q^{\prime}}=1$ if $q$ and $q^{\prime}$ belong to the same isospin doublet, and $\Delta_{q q^{\prime}}=0$ otherwise. The coefficients for the operators of type $V, A(\widetilde{V}, \widetilde{A})$, are determined by tree-level $Z(W)$ exchange and we neglect Cabibbo-Kobayashi-Maskawa angles.

The non-vanishing elements of the anomalous-dimension matrix for the operators in Eqs. (27)-(29) are

$$
\begin{gathered}
\gamma\left(V_{f}, V_{f}\right)=\frac{16}{3} Q_{f}^{2}\left(1+2 N_{f}\right) \\
\gamma\left(V_{f}, A_{f}\right)=12 Q_{f}^{2}
\end{gathered}
$$

$$
\gamma\left(A_{f}, V_{f}\right)=\frac{52}{3} Q_{f}^{2}
$$

$$
\gamma\left(V_{f f^{\prime}}, V_{f f^{\prime}}\right)=\frac{16}{3}\left(Q_{f}^{2} N_{f}+Q_{f^{\prime}}^{2} N_{f^{\prime}}\right)
$$

$$
\begin{gathered}
\gamma\left(V_{f f^{\prime}}, A_{f f^{\prime}}\right)=\gamma\left(A_{f f^{\prime}}, V_{f f^{\prime}}\right)=12 Q_{f} Q_{f^{\prime}} \\
\gamma\left(V_{f}, V_{f f^{\prime}}\right)=\frac{8}{3} Q_{f} Q_{f^{\prime}}\left(1+2 N_{f}\right)
\end{gathered}
$$

$$
\gamma\left(A_{f}, V_{f f^{\prime}}\right)=\frac{8}{3} Q_{f} Q_{f^{\prime}}
$$

$$
\begin{gathered}
\gamma\left(\widetilde{V}_{q q^{\prime}}, \widetilde{V}_{q q^{\prime}}\right)=\gamma\left(\widetilde{A}_{q q^{\prime}}, \widetilde{V}_{q q^{\prime}}\right)=12 Q_{q} Q_{q^{\prime}} \\
\gamma\left(\widetilde{V}_{q q^{\prime}}, V_{q}\right)=\gamma\left(\widetilde{A}_{q q^{\prime}}, V_{q}\right)=\frac{16}{3} Q_{q} Q_{q^{\prime}}
\end{gathered}
$$

$$
\begin{gathered}
\gamma\left(\widetilde{V}_{q q^{\prime}}, V_{q q^{\prime}}\right)=\gamma\left(\widetilde{A}_{q q^{\prime}}, V_{q q^{\prime}}\right)=\frac{8}{3}\left(Q_{q}^{2}+Q_{q^{\prime}}^{2}\right) \\
\gamma\left(\widetilde{V}_{q q^{\prime}}, V_{q f^{\prime \prime}}\right)=\gamma\left(\widetilde{A}_{q q^{\prime}}, V_{q f^{\prime \prime}}\right)=\frac{8}{3} Q_{q^{\prime}} Q_{f^{\prime \prime}},
\end{gathered}
$$

with $f^{\prime} \neq f, q^{\prime} \neq q$ and $f^{\prime \prime} \neq f, f^{\prime}$.

Inserting in Eq. (26) the Wilson coefficients at the scale $\mu=M_{Z}$ [see Eqs. (14)-(16) and Eqs. (30)-(32)], and the relevant elements of the anomalous-dimension matrix [see Eqs. (20)-(24) and Eqs. (33)-(45)], we obtain

$$
a_{\mu}^{\mathrm{EW}}(3 \text { loop })_{L L}=\frac{5 G_{\mu} m_{\mu}^{2}}{24 \sqrt{2} \pi^{2}}\left[\frac{\alpha\left(m_{\mu}\right)}{\pi}\right]^{2}(A+B) .
$$

Here $A$ and $B$ come respectively from the $(\gamma \gamma) / 2$ and the $b \gamma$ term in Eq. (26) and, in the approximation $s_{W}^{2}=1 / 4$ and $m_{s}$ $=m_{u}=m_{d}=m_{Q}$, are given by

$$
\begin{aligned}
A= & \frac{2827}{90} \ln ^{2} \frac{M_{Z}}{m_{\mu}}-\frac{298}{45} \ln ^{2} \frac{M_{Z}}{m_{\tau}}-\frac{7826}{3645} \ln ^{2} \frac{M_{Z}}{m_{b}}+\frac{7040}{729} \ln ^{2} \frac{M_{Z}}{m_{c}}+\frac{2108}{405} \ln ^{2} \frac{M_{Z}}{m_{Q}}+\frac{24}{5} \ln \frac{M_{Z}}{m_{b}} \ln \frac{M_{Z}}{m_{\mu}} \\
& +\frac{72}{5} \ln \frac{M_{Z}}{m_{\tau}} \ln \frac{M_{Z}}{m_{\mu}}-\frac{96}{5} \ln \frac{M_{Z}}{m_{c}} \ln \frac{M_{Z}}{m_{\mu}}-\frac{48}{5} \ln \frac{M_{Z}}{m_{Q}} \ln \frac{M_{Z}}{m_{\mu}}-\frac{128}{1215} \ln \frac{M_{Z}}{m_{b}} \ln \frac{M_{Z}}{m_{c}}
\end{aligned}
$$




$$
\begin{aligned}
B= & -\frac{179}{45}\left(\frac{1}{3} \ln ^{2} \frac{M_{Z}}{m_{b}}+\ln ^{2} \frac{M_{Z}}{m_{\tau}}+\frac{4}{3} \ln ^{2} \frac{M_{Z}}{m_{c}}+2 \ln ^{2} \frac{M_{Z}}{m_{Q}}+2 \ln ^{2} \frac{M_{Z}}{m_{\mu}}\right)+\frac{2}{5}\left(\ln ^{2} \frac{m_{b}}{m_{\tau}}+\frac{4}{3} \ln ^{2} \frac{m_{b}}{m_{c}}+2 \ln ^{2} \frac{m_{b}}{m_{Q}}+2 \ln ^{2} \frac{m_{b}}{m_{\mu}}\right) \\
& -\frac{8}{5}\left(2 \ln ^{2} \frac{m_{c}}{m_{Q}}+2 \ln ^{2} \frac{m_{c}}{m_{\mu}}\right)+\frac{6}{5}\left(\frac{4}{3} \ln ^{2} \frac{m_{\tau}}{m_{c}}+2 \ln ^{2} \frac{m_{\tau}}{m_{Q}}+2 \ln ^{2} \frac{m_{\tau}}{m_{\mu}}\right)-\frac{8}{5} \ln ^{2} \frac{m_{Q}}{m_{\mu}} .
\end{aligned}
$$

Using the same values for the quark masses as in Sec. II, we find

$$
\frac{a_{\mu}^{\mathrm{EW}}(3 \text { loop })_{L L}}{a_{\mu}^{\mathrm{EW}}(2 \text { loop })_{L L}} \simeq-0.8 \frac{\alpha}{\pi} \ln \frac{M_{Z}}{m_{\mu}}
$$

which corresponds to a $1 \%$ reduction of $a_{\mu}^{\mathrm{EW}}(2$ loop) and gives $a_{\mu}^{\mathrm{EW}}(3 \text { loop })_{L L}=0.5 \times 10^{-11}$. Including all the different contributions, we obtain

$$
a_{\mu}^{\mathrm{EW}}=(153 \pm 3) \times 10^{-11} .
$$

\section{NEW PHYSICS EFFECTS IN $a_{\mu}^{\text {EW }}$ AND IN THE ELECTRON EDM}

The effective-Lagrangian method is well suited to discuss effects from new physics. Indeed, in the presence of new interactions, characterized by a mass scale $\Lambda_{N P}$ of the order of the weak scale or larger, only the ultraviolet behavior of the theory is modified, while the infrared one is unaffected. This means that information about new physics can be completely included in the matching conditions of the Wilson coefficients, while the QED renormalization described by the anomalous dimension matrix through Eq. (17) remains the same.

Furthermore, it is reasonable to assume that new physics has a chance to affect sizably only matching conditions of operators that, in the SM, are not generated at the tree level, but possibly at the quantum level. This is indeed what happens in most of the SM extensions generally considered. In this case, the analysis is particularly simple and predictive.

Let us first consider new physics contributions to $a_{\mu}$. Following our assumption, we expect that only the matching condition of the operator $H_{\mu}$ is affected, but not those of the four-fermion operators $V$ and $A$. This means that the total $a_{\mu}$ is just given by the sum of the SM result discussed in the previous section and of a new contribution $a_{\mu}^{\mathrm{NP}}$. If $a_{\mu}^{\mathrm{NP}}$ is known at one loop, the leading contribution at two loops, enhanced by large QED logarithms, can be simply obtained:

$$
a_{\mu}^{\mathrm{NP}}(2 \text { loop })=-\frac{4 \alpha}{\pi} \ln \frac{\Lambda_{N P}}{m_{\mu}} a_{\mu}^{\mathrm{NP}}(1 \text { loop }) .
$$

The coefficient in Eq. (51) corresponds to the anomalous dimension element $\gamma\left(H_{\mu}, H_{\mu}\right)$. For instance, for $\Lambda_{N P}$ $=100 \mathrm{GeV}$, the inclusion of the two-loop contribution reduces the one-loop result by $6 \%$. This result is quite model independent, and it can be applied to specific models in which $a_{\mu}^{\mathrm{NP}}$ (1 loop) is known, as in the case of supersymmetry [16], light-gravitino interactions [17], compositeness [18], leptoquarks [19], and light non-minimal Higgs bosons [20].

Another case in which the QED logarithms turn out to be large is represented by the EDM of the electron, $d_{e}$. In the $\mathrm{SM}, d_{e}$ is negligible, but in some of its extensions with new sources of $C P$ violation, it can lie just below the present experimental upper bound. For instance, this can be the case in some versions of the supersymmetric model [21].

The analysis of the QED renormalization can be done along the same lines followed for $a_{\mu}$. The electron EDM corresponds to an operator in the effective Lagrangian:

$$
-\frac{i}{2} d_{e} \bar{e} \sigma_{\nu \rho} \gamma_{5} e F^{\nu \rho}
$$

It is easy to show that in the effective theory below the weak scale, its one-loop anomalous dimension is equal to that of the magnetic dipole operator that is given in Eq. (20). Therefore, if $d_{e}^{(0)}$ describes the new physics contribution obtained by integrating out the heavy modes with mass $\Lambda_{N P}$, the QED improved result is

$$
d_{e}=d_{e}^{(0)}\left[1-\frac{4 \alpha}{\pi} \ln \frac{\Lambda_{N P}}{m_{e}}\right]
$$

For $\Lambda_{N P}=100 \mathrm{GeV}$, the term inside brackets amounts to a reduction of $d_{e}^{(0)}$ of $11 \%$.

The result in Eq. (53) is rather model independent. However, it should be remarked that it is valid only if $\Lambda_{N P}$ is not much larger than the weak scale. An analysis at very large scales should include the mixing of the operator in Eq. (52) with other $C P$ violating operators, like the analogue of Eq. (52) for the different electroweak gauge bosons, and the analogue of the Weinberg operator [22] for the SU(2) gauge theory. The corresponding anomalous dimension matrix can be extracted from Ref. [23]. The result, however, is more involved than the one presented in Eq. (53), since it depends on the separate unknown coefficients of the different operators.

\section{ACKNOWLEDGMENTS}

We wish to thank G. Altarelli and M. Ciuchini for useful discussions. 
[1] Particle Data Group, L. Montanet et al., Phys. Rev. D 50, 1173 (1994).

[2] For a review, see T. Kinoshita and W. J. Marciano, in Quantum Electrodynamics, edited by T. Kinoshita (World Scientific, Singapore, 1990), p. 419, and references therein.

[3] G. Bunce, as quoted in Ref. [11].

[4] M. Davier and A. Höcker, hep-ph/9801361.

[5] B. Krause, Phys. Lett. B 390, 392 (1997).

[6] M. Hayakawa and T. Kinoshita, Phys. Rev. D 57, 465 (1998).

[7] K. Fujikawa, B. W. Lee, and A. I. Sanda, Phys. Rev. D 6, 2923 (1972); R. Jackiw and S. Weinberg, ibid. 5, 2473 (1972); G. Altarelli, N. Cabibbo, and L. Maiani, Phys. Lett. 40B, 415 (1972); I. Bars and M. Yoshimura, Phys. Rev. D 6, 374 (1972); W. A. Bardeen, R. Gastmans, and B. E. Lautrup, Nucl. Phys. B46, 315 (1972).

[8] T. V. Kukhto, E. A. Kuraev, A. Schiller, and Z. K. Silagadze, Nucl. Phys. B371, 567 (1992).

[9] A. Czarnecki, B. Krause, and W. J. Marciano, Phys. Rev. D 52, 2619 (1995).

[10] E. de Rafael, S. Peris, and M. Perrottet, Phys. Lett. B 355, 523 (1995)

[11] A. Czarnecki, B. Krause, and W. J. Marciano, Phys. Rev. Lett. 76, 3267 (1996).

[12] For a review, see G. Buchalla, A. J. Buras, and M. E. Lautenbacher, Rev. Mod. Phys. 68, 1125 (1996) and references therein.

[13] M. Ciuchini, E. Franco, L. Reina, and L. Silvestrini, Nucl. Phys. B421, 41 (1994).

[14] G. 't Hooft and M. Veltman, Nucl. Phys. B44, 189 (1972).

[15] W. Siegel, Phys. Lett. 84B, 193 (1979).
[16] P. Fayet, in Unification of the Fundamental Particle Interactions, edited by S. Ferrara, J. Ellis, and P. van Nieuwenhuizen (Plenum, New York, 1980), p. 587; J. A. Grifols and A. Mendez, Phys. Rev. D 26, 1809 (1982); J. Ellis, J. S. Hagelin, and D. V. Nanopoulos, Phys. Lett. 116B, 283 (1982); R. Barbieri and L. Maiani, ibid. 117B, 203 (1982); D. A. Kosower, L. M. Krauss, and N. Sakai, ibid. 133B, 305 (1983); T. C. Yuan, R. Arnowitt, A. H. Chamseddine, and P. Nath, Z. Phys. C 26, 407 (1984); J. Lopez, D. V. Nanopoulos, and X. Wang, Phys. Rev. D 49, 366 (1991); U. Chattopadhyay and P. Nath, ibid. 53, 1648 (1996); T. Moroi, ibid. 53, 6565 (1996); M. Carena, G. F. Giudice, and C. E. M. Wagner, Phys. Lett. B 390, 234 (1997).

[17] A. Mendez and F. X. Orteu, Nucl. Phys. B256, 181 (1985); T. Li, J. L. Lopez, and D. V. Nanopoulos, hep-ph/9704439; F. Ferrer and J. A. Grifols, Phys. Rev. D 56, 7466 (1997).

[18] S. Brodsky and S. Drell, Phys. Rev. D 22, 2236 (1980).

[19] G. Couture and H. König, Phys. Rev. D 53, 555 (1996).

[20] M. Krawczyk and J. Zochowski, Phys. Rev. D 55, 6968 (1997).

[21] For a review, see Y. Grossman, Y. Nir, and R. Rattazzi, in Heavy Flavours II, edited by A. J. Buras and M. Lindner, Advanced Series on Directions in High Energy Physics (World Scientific, Singapore, in press), hep-ph/9701231. For recent analyses, see Y. Kizukuri and N. Ohimo, Phys. Rev. D 46, 3025 (1992); T. Falk and K. A. Olive, Phys. Lett. B 375, 196 (1996); T. Ibrahim and P. Nath, Phys. Rev. D 57, 478 (1998).

[22] S. Weinberg, Phys. Rev. Lett. 63, 2333 (1989).

[23] E. Braaten, C. S. Li, and T. C. Yuan, Phys. Rev. Lett. 64, 1709 (1990). 